\title{
FATORES ASSOCIADOS À FUNÇÃO SEXUAL DE MULHERES IDOSAS
}

\author{
Juliana Cordeiro Carvalho, ' Rogério Dubosselard Zimmermann, ${ }^{2}$ Maria da Conceição Lafayette de Almeida, ${ }^{3}$ \\ Suelane Renata de Andrade Silva ${ }^{4}$ e Monique de Freitas Gonçalves Lima ${ }^{5}$
}

FACTORS ASSOCIATED WITH THE SEXUAL FUNCTION OF ELDERLY WOMEN

\section{FACTORES ASOCIADOS A LA FUNCIÓN SEXUAL DE LAS MUJERES MAYOR}

\begin{abstract}
Resumo: Avaliar os fatores associados à função sexual de mulheres idosas. Método: trata-se de um estudo observacional-descritivo, com abordagem quantitativa, de corte transversal. Foram avaliadas II 0 idosas cadastradas nos cursos de línguas pela Universidade Aberta da Terceira Idade (UNATI) inserida na Universidade Federal de Pernambuco (UFPE), em Recife-PE. Os dados foram coletados por meio do instrumento Quociente Sexual Feminino (QSF) e questionário estruturado, sendo posteriormente analisados por estatística descritiva e inferencial. Resultados: a função sexual foi de nulo a ruim 50 (45,5\%) e esteve associada com arranjo familiar $(0,033)$, estado civil $(0,020)$, filhos $(0,003)$, etilismo $(0,028)$. Conclusão: a função sexual foi influenciada por morar com familiares, não ter companheiro(a) e ter filhos.
\end{abstract}

Palavras-chaves: Sexualidade; saúde da pessoa idosa; fatores associados.

\begin{abstract}
To evaluate the factors associated with the sexual function of elderly women. Method: This is an observational-descriptive study, with a quantitative, cross-sectional approach. 110 elderly women registered in language courses by the University were evaluated. Open for Seniors (UNATI) inserted at UFPE / Recife-PE Universidade Federal de Pernambuco (UFPE). Data were collected using the Female Sexual Quotient (QSF) instrument and a structured questionnaire, which were subsequently analyzed using descriptive and inferential statistics. Results: The sexual function was null to bad $50(45,5 \%)$ and was associated with family arrangement $(0,033)$, marital status $(0,020)$, children $(0,003)$, alcoholism $(0,028)$. Conclusion: Sexual function was influenced by living with family members, not having a partner and having children.
\end{abstract}

Keywords: Sexuality; Health of the Elderly; Associated Factors.

Resumen: Evaluar factores asociados a la función sexual en mujeres mayores. Método: Se trata de un estudio observacional-descriptivo, con abordaje cuantitativo y transversal. Se evaluaron 110 ancianas inscritas en cursos de idiomas en la Universidad. Abierto de Ciudadanos Mayores (UNATI) en Universidade Federal de Pernambuco (UFPE), em Recife-PE. Los datos fueron recolectados utilizando el instrumento de Cociente Sexual Femenino (QSF) y un cuestionario estructurado, y luego analizados mediante estadística descriptiva e inferencial. Resultados: La función sexual varió de nula a pobre $50(45,5 \%)$ y se asoció con arreglo familiar $(0,033)$, estado civil $(0,020)$, hijos $(0,003)$, alcoholismo $(0,028)$. Conclusión: La función sexual se vio influenciada por vivir con miembros de la familia, no tener pareja y tener hijos.

Palabras clave: Sexualidad; Salud de los Ancianos; Factores Asociados.

\footnotetext{
Doutoranda em Gerontologia pela Universidade Estadual de Campinas (Unicamp), mestra em Gerontologia pelo Programa de Pós-Graduação em Gerontologia (PPGERO) da Universidade Federal de Pernambuco (UFPE) e fisioterapeuta. E-mail: julianacordeirocarvalho89@gmail.com

2 Docente pelo Programa de Pós-Graduação em Gerontologia (PPGERO) da Universidade Federal de Pernambuco (UFPE). E-mail: rdzlegal@gmail.com

${ }^{3}$ Docente pelo Programa de Pós-Graduação em Gerontologia (PPGERO) da Universidade Federal de Pernambuco (UFPE). E-mail: celafayette@ hotmail.com

${ }^{4}$ Doutoranda em Enfermagem pela Universidade Federal da Paraíba (UFPB), mestra em Gerontologia pelo Programa de Pós-Graduação em Gerontologia (PPGERO) da Universidade Federal de Pernambuco (UFPE) e nutricionista. E-mail: suelanerenata@yahoo.com.br

${ }^{5}$ Doutoranda em Enfermagem pela Universidade Federal de Pernambuco (UFPE), mestra em Gerontologia pelo Programa de Pós-Graduação em Gerontologia (PPGERO) da Universidade Federal de Pernambuco (UFPE) e enfermeira. E-mail: monique_freitas@hotmail.com
} 


\section{Introdução}

Pessoas mais velhas ainda são rotuladas pela sociedade, que não podem e nem devem sentir vontade de ter relação sexual (AIZENBERG et al., 2002; SIQUEIRA, 2017). Esse pensamento negativo está relacionado ao modelo de sexualidade estabelecido como "normal" e é centrado no culto ao corpo, à juventude, à beleza, na consideração do sexo como fonte de prazer restrito ao coito e para fins de procriação (AIZENBERG et al., 2002).

Devemos lembrar que a velhice não é sinônimo de incapacidade motora ou ausência de vivências sociais e sexuais (VIEIRA et al., 20I4). Mesmo com as limitações da idade é possível supor que os(as) idosos(as) possam ter momentos ou relacionamentos afetivos e sexuais (COSTA et al., 2017).

Para a Organização Mundial de Saúde (2019), a sexualidade organiza "uma energia que nos motiva para encontrar amor, contato, ternura e intimidade" e está associada ao nosso modo de agir, sentir e de se relacionar com o outro.

As pessoas idosas que conseguem entender e aceitar o processo de envelhecimento e a chegada da velhice vivenciam a sexualidade de forma mais saudável e satisfatória. A compreensão sobre este processo se faz importante na tentativa de minimizar o julgamento de que os(as) idosos(as) são impotentes e incapazes de desfrutar de sua sexualidade apenas por pertencerem à faixa etária dos 60 anos ou mais (ALENCAR et al., 20I4).

Sabe-se que a sexualidade é impossível de ser abordada de forma isolada por receber influência dos fatores biológicos, sociais, culturais, psicológicos, econômicos e religiosos (VIEIRA et al., 2014).

Outro ponto que deve se destacar é a feminização da velhice, as mulheres idosas brasileiras têm apresentado maior expectativa de vida, devido à ação conjunta de três fatores: a ampliação da cobertura previdenciária, o maior acesso aos serviços de saúde e o crescimento da tecnologia médica, o que representa uma melhoria de suas condições de vida (CAMARANO, 2003).

Campos (2014) acrescenta que a menor mortalidade feminina explica essa diferença, bem como os comportamentos específicos do homem e da mulher: mulheres frequentam mais os centros de saúde, homens estão mais expostos a acidentes de trabalho e de trânsito e somam-se à prevalência de alcoolismo, drogas e tabagismo - vícios que afetam também mulheres, mas em menor proporção. As mudanças que acompanham essa etapa de vida são predominantemente femininas, pode-se dizer que a velhice se feminizou (SALGADO, 2002).

Dessa forma, o objetivo da pesquisa foi avaliar os fatores que interferem na função sexual de mulheres idosas.

\section{Método}

Trata-se de um estudo observacional-descritivo, quantitativo de corte transversal. A pesquisa foi realizada na Universidade Aberta à Terceira Idade (UNATI), localizada no campus da Universidade Federal de Pernambuco (UFPE).

A amostra, do tipo censitária, incluiu 120 pessoas, com idade igual ou superior a 60 anos, do sexo feminino, que frequentaram os cursos de línguas (inglês, espanhol e italiano) entre março e junho de 2019. Foram excluídas idosas que tinham algum déficit cognitivo, deficiência auditiva e as que não concordaram em participar do estudo. Ao final, a amostra resultou em 1 I 0 mulheres idosas.

Inicialmente foi realizada uma apresentação sobre o projeto às idosas, abordando o tema sexualidade durante a velhice. Para este fim, foi utilizado um vídeo disponível no canal do YouTube cujo título é: "Profissão Repórter 13/12/2017 - Sexo na Terceira Idade" que retratava a vivência da sexualidade no público idoso. Esse recurso tinha $\circ$ objetivo de deixá-las mais à vontade e, consequentemente, propensas a responderem sobre a temática. Os dados foram coletados por meio de entrevista com questionário estruturado composto pelas variáveis arranjo familiar, estado civil, filhos, renda mensal, anos de estudo, etilismo, tabagismo, hipertensão arterial, diabetes e a imagem corporal pela escala nove Silhuetas, proposta por Sorensen e Stunkard (PEREIRA et al., 2016).

A escala de nove Silhuetas proposta por Sorensen e Stunkard foi utilizada com o intuito de avaliar a imagem corporal dessas mulheres. É constituída por desenhos de nove silhuetas com ordem gradativa crescente do tamanho corporal. Para verificar a satisfação ou a insatisfação com a imagem corporal deve-se calcular a diferença entre a silhueta real e a silhueta ideal. Se estiver satisfeito com a sua aparência (variação igual à zero), insatisfeito com a sua aparência (variação diferente de zero). Quando a diferença for positiva, considera-se insatisfatória por excesso de peso e quando for negativa, insatisfatória por magreza. As idosas marcavam o corpo do desenho que representavam a sua aparência física atual e a que gostaria de ter (PEREIRA et al., 2016).

O desempenho/satisfação sexual (função sexual) das mulheres foi avaliado pelo Quociente Sexual Feminino (QS-F), nos últimos seis meses, onde o resultado do padrão de desempenho sexual foi dividido na seguinte pontuação: 82 a I 00 - Bom a Excelente; 42 a 80 - Regular; e 22 a 40 - Nulo a Ruim (FLEURY et al., 20।7).

O QS-F compõe-se de dez questões, cada qual devendo ser respondida em uma escala do tipo likert, na qual o "0" representa nunca, "I" = raramente, " 2 " = às vezes, " 3 " = aproximadamente $50 \%$ das vezes, " 4 " = a maioria das vezes, " 5 " = sempre. No final essas questões foram agrupadas e avaliados os seguintes aspectos: 
desejo e interesse sexual (questões I,2,8); preliminares (questão 3); excitação da mulher e sintonia com o parceiro (questões 4,5); conforto na relação sexual (questões 6,7); orgasmo e satisfação sexual (questões 9,10).

Os dados foram digitados com dupla entrada no software EPI-INFO versão 3.3.2. As análises dos dados foram descritas em frequências absolutas, porcentagens, médias e desvios-padrão.

Para verificar possíveis associações entre as variáveis categóricas, foi utilizado o teste do qui-quadrado e o exato de Fisher. Todas as análises foram realizadas no software Statistical Package for the Social Sciences (SPSS) para Windows, versão I3.0.

O estudo foi aprovado pelo Comitê de Ética em Pesquisas do Centro de Ciências da Saúde da Universidade Federal de Pernambuco, sob o número de registro CAAE $0|4365|$ I .1 .0000 .5208 . Todas as entrevistadas foram previamente informadas dos objetivos da pesquisa e, após o consentimento, ocorreu a assinatura no Termo de Consentimento Livre e Esclarecido (TCLE).

\section{Resultados}

Das 110 idosas entrevistadas 50 (45,5\%) apresentaram desempenho/satisfação sexual de nulo a ruim, 33 (40,0\%) regular e 27 (14,5\%) de bom a excelente. A idade variou entre 60 e 84 anos, com média de 67, I e o desvio padrão foi de 5,25.

Do total, 76 (69\%) moravam com familiares, 75 (68,2\%) referiram não possuir companheiro(a), 94 $(85,5 \%)$ tinham filhos, $102(92,7 \%)$ tinham renda maior que um salário mínimo, $64(58,1 \%)$ tinham mais que 12 anos de estudos, 106 (96,4\%) não ingeriam bebidas alcoólicas, 106 (96,4\%) não fumavam (tabaco), 56 (50,9\%) não possuíam hipertensão arterial, $8 \mathrm{I}(73,6 \%)$ não eram diabéticas, 69 (6I,8\%), não eram cardiopatas, 102 (92,7\%) faziam uso de algum medicamento e $88(80 \%)$ desejavam ter um corpo magro.

Em relação aos fatores associados à função sexual, foram observadas relações estatisticamente significativas com o arranjo familiar, estado civil, ter filhos e etilismo (Tabela I).

Tabela I - Função Sexual segundo as características das idosas frequentadoras da UNATI/ UFPE. Recife, PE, 20I9

\begin{tabular}{|c|c|c|c|c|c|}
\hline \multirow{2}{*}{ Características (n) } & \multirow{2}{*}{ n (\%) } & \multicolumn{3}{|c|}{ Quociente Sexual Feminino (QSF) } & \multirow{2}{*}{ p-valor } \\
\hline & & Bom a Excelente & Regular & Nulo a Ruim & \\
\hline Arranjo Familiar & & $27(24,5)$ & $33(30)$ & $50(45,5)$ & $0,033^{\mathrm{a}}$ \\
\hline Com companheiro (a) & $21(19)$ & $7(33,3)$ & II (I0) & $3(2,7)$ & \\
\hline Sozinha & $34(3 \mathrm{I})$ & $7(20,5)$ & $12(35,2)$ & $15(44,1)$ & \\
\hline Com outros familiares & $55(50)$ & I3 $(11,8)$ & $10(9)$ & $32(29)$ & \\
\hline Estado Civil & & & & & $0,020^{\mathrm{a}}$ \\
\hline Com companheiro(a) & $35(31,8)$ & II $(3 \mid, 4)$ & $16(45,7)$ & $8(22,8)$ & \\
\hline Sem companheiro(a) & $75(68,2)$ & $16(2 \mid, 3)$ & $17(22,66)$ & 42 (56\%) & \\
\hline Filhos & & & & & $0,003^{a}$ \\
\hline Sim & $94(85,5)$ & $25(26,5)$ & $25(26,5)$ & $44(46,8)$ & \\
\hline Não & $16(14,5)$ & $2(12,5)$ & $8(50)$ & $6(37,5)$ & \\
\hline Renda Mensal (SM)* & & & & & $0,330^{a}$ \\
\hline$<1$ & $8(7,2)$ & $2(1,8)$ & $2(1,8)$ & $4(3,6)$ & \\
\hline$>1$ & $102(92,7)$ & $25(22,7)$ & $31(28, I)$ & $46(4 I, 8)$ & \\
\hline Anos de Estudo & & & & & $0,340^{a}$ \\
\hline$\leq 12$ & $46(4 I, 8)$ & $8(7,2)$ & $12(10,9)$ & $26(23,6)$ & \\
\hline$>12$ & $64(58,1)$ & $19(17,2)$ & $21(19)$ & $24(21,8)$ & \\
\hline Etilismo & & & & & $0,028^{a}$ \\
\hline Sim & $4(3,6)$ & 0 & $4(100)$ & 0 & \\
\hline Não & $106(96,4)$ & $27(25,4)$ & $29(27,3)$ & $50(47, I)$ & \\
\hline
\end{tabular}


20 Revista Brasileira de

\begin{tabular}{|c|c|c|c|c|c|}
\hline & & & & & (continua \\
\hline Tabagismo & & & & & $0,704^{\mathrm{a}}$ \\
\hline Sim & $4(3,6)$ & I $(0,9)$ & $2(1,8)$ & $I(0,9)$ & \\
\hline Não & $106(96,4)$ & $26(23,6)$ & $31(28,1)$ & $49(44,5)$ & \\
\hline Hipertensão Arterial & & & & & $0,109^{b}$ \\
\hline Sim & $54(49,1)$ & $13(1 \mid, 8)$ & II $(12,7)$ & $30(27,2)$ & \\
\hline Não & $56(50,9)$ & $14(12,7)$ & $22(20)$ & $20(18,1)$ & \\
\hline Diabetes & & & & & $0,283^{b}$ \\
\hline Sim & $29(26,4)$ & $10(9)$ & $5(4,5)$ & $14(12,7)$ & \\
\hline Não & $81(73,6)$ & $17(15,4)$ & $28(25,4)$ & $36(32,7)$ & \\
\hline Cardiopatias & & & & & 0,276 \\
\hline Sim & $8(7,3)$ & $6(5,4)$ & $0(0)$ & $2(1,8)$ & \\
\hline Não & $102(92,7)$ & $44(40)$ & $33(30)$ & $25(22,7)$ & \\
\hline Medicação & & & & & 0,288 \\
\hline Sim & $88(80)$ & $42(38, I)$ & $24(21,8)$ & $22(20)$ & \\
\hline Não & $22(20)$ & $8(7,2)$ & $9(8,1)$ & $5(4,5)$ & \\
\hline Imagem Corporal & & & & & $0,8 \mid 4^{a}$ \\
\hline Satisfação Corporal & $35(31,8)$ & $9(25,7)$ & $10(28,5)$ & $16(45,7)$ & \\
\hline Ter um corpo maior & $7(6,4)$ & $2(28,5)$ & $2(28,5)$ & $3(42,8)$ & \\
\hline Ter um corpo magro & $69(61,8)$ & $16(23,1)$ & $21(30,4)$ & $31(44,9)$ & \\
\hline
\end{tabular}

*salário-mínimo vigente no ano de 2019; ${ }^{\mathrm{p}}$-valor do teste Exato de Fisher para comparação de proporção ${ }^{\mathrm{b}} \mathrm{p}$-valor do teste do qui-quadrado para comparação de proporção.

\section{Discussão}

A sexualidade é um componente importante da intimidade emocional e física que a maioria das pessoas deseja experimentar ao longo da vida (CARDOSO et al., 2016). Os fatores que podem interferir no desempenho/ satisfação sexual decorrem de aspectos individuais, fisiológicos e sociais, das limitações que podem ocorrer na velhice. As dificuldades na aceitação da atividade sexual nessa fase podem advir tanto pela ausência de informação como na conceituação que a sexualidade esteja restrita à genitália e ao ato sexual (ALENCAR et al., 20I4).

Neste estudo, o desempenho/satisfação ou função sexual das idosas mostrou-se de nulo a ruim (45,5\%), regular (30\%) e bom a excelente (24,5\%). Pesquisa realizada em grupos da terceira idade no município de Campo Mourão (PR) revela que das 33 mulheres idosas, 19 (50\%) apresentaram função sexual regular (POLIZERA; ALVES, 2009). A diferença dos resultados pode estar relacionada com fatores biopsicossociais (VIEIRA et al., 20I4), mas percebe-se que os resultados não foram totalmente distintos, havendo um comprometimento da função sexual em ambos os estudos. O prejuízo na função sexual das idosas também pode estar associado à visão cultural sobre a idosa que pode interferir na manutenção da vida sexual, ao se sentirem vítimas de preconceito sexual devido à idade (RODRIGUES et al., 20I8).

A sexualidade não é expressa somente pelo ato sexual. Àmedida que o corpo não responde mais ao desejo, as adaptações sexuais se tornam necessárias e ajudam na expressão da sexualidade em idosos (ALENCAR et al., 2014).

Sabe-se que a experiência sexual feminina apresenta uma enorme diversidade em qualquer fase da vida, algumas não têm mais parceiro sexual, outras vivenciam sexo solitário ou mantêm relacionamento afetivo, acompanhado ou não de atividade sexual. ${ }^{12}$ Relatos de uma população entre 80 e 102 anos revelam que as atividades mais frequentes nessa faixa etária são: tocar e acariciar sem coito, masturbação e, por último, intercurso sexual (NASCIMENTO, 20I6).

Um dos fatores que estiveram associados à função sexual nas idosas neste estudo foi o fato de morar com outros familiares, correspondendo a 50\%. A falta de 
privacidade dos idosos para a prática sexual também esteve presente em outro estudo em que mostrou que $51,7 \%$ das idosas eram ativas sexualmente, porém relataram que existiam fatores que influenciavam na sexualidade como a indisposição, a falta de interesse, problemas de saúde, privacidade e disponibilidade de companheiro(a) (SOARES, 2009).

Com os resultados obtidos, percebeu-se que as idosas que tinham filhos obtiveram uma melhor função sexual. Sabe-se que com a valorização do corpo, a proliferação de literatura sobre higiene e proteção, os métodos para ter filhos saudáveis, contribuíram para uma vivência da sexualidade mais segura e liberal, evitando assim que as mulheres idosas adoecessem, prejudicando a sua qualidade de vida e influenciando na sua vivência sexual (FERREIRA, 20I0).

Além disso, as famílias conservadoras geralmente não aceitam as necessidades sexuais de idosos divorciados ou viúvos, contribuindo para os sentimentos negativos dos idosos sobre si mesmos (LINDAU; GAVRILOVA, 20I0).

Outro fator foi a falta de companheiro relatada por $68,2 \%$ das idosas e destas, a maioria obteve uma função sexual de nulo a ruim, mostrando que a falta de companheiro pode ter interferência na função sexual. Percebe-se, na literatura, que o fato de morar com o(a) esposo(a), gera uma melhora na qualidade de vida, em que foi avaliada pela escala de WHOQOL-100, ao considerar que há maior afetividade, companheirismo, corresponsabilização do cuidado e maior regularidade e satisfação nas relações sexuais desses indivíduos em que utilizaram a escala do Quociente Sexual Feminino (QSF) (ABDO, 20I6; PIZZOLATTO, 2018; SATHYANARAYANA; ABHINAV, 2018).

Outros estudos afirmam que mesmo com a ausência de um parceiro, a busca pelo prazer pode ser obtida por outras formas. Os fatores que podem afetar a expressão da sexualidade ou no ato sexual estão relacionados aos aspectos individuais, fisiológicos e sociais, e, mesmo com algumas limitações que podem ocorrer durante a velhice, a satisfação sexual ainda pode permanecer (HOWARD et al., 2006).

Dentro desse contexto, é importante a orientação sexual quanto às modificações corporais e fisiológicas que podem interferir na sexualidade dessa população, assim como a influência cultural que ainda permeia, de que o idoso é um ser assexual. Dessa forma, destaca-se a importância dos profissionais de saúde na identificação desses fatores durante a anamnese, a fim de contribuir, por meio de atividades educativas para desmistificar 0 preconceito que persiste na sexualidade durante a velhice (RODRIGUES et al., 2019).

\section{Conclusão}

O estudo evidenciou que a função sexual das idosas foi de nulo a ruim, em sua grande maioria, e tiveram associação pelo fato de morar com familiares, não ter companheiro(a), ter filhos e ingerir bebidas alcoólicas de forma moderada.

É relevante identificar os fatores que influenciam na sexualidade de mulheres idosas, para evitar o surgimento de um comprometimento da função sexual. É de suma importância realizar atividades educativas, para desmistificação da temática, cessando os mitos e tabus e assim poder melhorar a sexualidade.

Espera-se que esse estudo mostre para a população que existem inúmeros fatores que podem influenciar na função sexual de mulheres idosas e a importância de identificá-los para assim melhorar a qualidade de vida deste público.

Sugere-se a realização de novas pesquisas que avaliem a função e satisfação sexual em mulheres idosas com outros níveis de escolaridade, evitando assim o viés sobre o nível sociocultural, já que elas frequentavam a Universidade Aberta para a Terceira Idade e pelo interesse em participar de um curso de línguas estrangeiras.

\section{Referências}

ABDO, C. H. N. Elaboração e validação do Quociente Sexual - versão feminina. Revista Brasileira de Medicina, Rio de Janeiro, v. 63, n. 9 , p. $477-487,2016$.

AIZENBERG, D. et al. Attitudes Toward Sexuality Among Nursing Home Residents. Sexuality and Disability, v. 20, n. 3, p. I85-189, 2002.

ALENCAR, D. L. et al. Fatores que interferem na sexualidade de idosos: uma revisão integrativa. Ciência \& Saúde Coletiva, Rio de Janeiro, v. 19, n. 8, p. 3533-3542, 2014.

ANDRADE, P. B. S.; BENITO, L. A. O. Perfil da sexualidade de pessoas idosas portadoras de SIDA/AIDS atendidas em um serviço de saúde do Distrito Federal. Universitas: Ciências da Saúde, Brasília, v. I4, n. 2, p. I05-II3, jul./dez. 2016.

CAMARANO, A. A. Mulher idosa: suporte familiar ou agente de mudança. Estudos Avançados, São Paulo, v. 17, n. 49, dez. 2003. 
CAMPOS, A. C. V. Envelhecimento, gênero e qualidade de vida. 20I4. Tese (Doutorado em Odontologia - área de concentração em Saúde Coletiva) - Universidade Federal de Minas Gerais, Belo Horizonte, 2014.

CARDOSO, F. L. et al. Da juventude à velhice: sexualidade de idosos praticantes de atividade física. Arquivos Catarinenses de Medicina, Florianópolis, v. 4I, n. I, p. 34-40, 2016.

CAVALCANTI, I. F. et al. Função sexual e fatores associados à disfunção sexual em mulheres no climatério. Revista Brasileira de Ginecologia e Obstetrícia, Rio de Janeiro, v. 36, n. II, p. 497-502, 2014.

COSTA, D. C. A. et al. Sexualidade no idoso: percepção de profissionais da geriatria e gerontologia. Universitas: Ciências da Saúde, Brasília, v. I5, n. 2, p. 75-80, jul./dez. 2017.

GRANDIM, C. V. C. et al. A prática sexual e o envelhecimento. Cogitare Enfermagem, Curitiba, v. I2, n. 2, p. 204-2I3, 2007.

FERNANDES, M. G. M. et al. Prevalência e determinantes de sintomas depressivos em idosos atendidos na atenção primária de saúde. Revista RENE, Fortaleza, v. II, n. I, p. 19-27, 2010.

FERREIRA, P. M. Sexualidades em Portugal: comportamentos e riscos. Lisboa: Editorial Bizânico, 2010. p. 55-104.

FLEURY, H. J. et al. Sexualidade da mulher idosa. Diagnóstico Tratamento, São Paulo, v. 20, n. 3, p. II7-I20, 2017.

HOWARD, J. R. et al. Factor saffecting sexuality in older Australian women: sexual interest, sexual arousal, relationship sand sexual distress in older Australian women. Climacteric: The Journal of The International Menopause Society, v. 9, n. 5, p. 355-67, 2006.

LINDAU, S. T.; GAVRILOVA, N. Sex, Health, and Years of sexually active life gained due to good health: evidence from two US population based cross sectional surveys of ageing. BMJ, v. 340 , n. $810,2010$.

MARTINS, J. J. et al. O cuidado no contexto domiciliar: o discurso de idosos/familiares e profissionais. Revista Enfermagem UERJ, v. I7, p. 556-562, 2009.
NASCIMENTO, R. F. Vivência da sexualidade pelas mulheres idosas. 2016. Dissertação (Mestrado Acadêmico em Saúde e Envelhecimento) Faculdade de Medicina de Marília, 2016.

ORGANIZAÇÃO MUNDIAL DA SAÚDE. Relatório Mundial de Envelhecimento e Saúde. [S. I.: s. n.], 2019.

PEREIRA, V. V. et al. Disfunção sexual e o uso de drogas: uma análise diagnóstica. Arquivos de Ciências da Saúde UNIPAR, Umuarama, v. 20, n. 2, p. 89-94, 2016.

PIZZOLATTO, C. E. A sala de aula de língua estrangeira com adultos da terceira idade. In: ROCHA, H. C.; BASSO, A. E. Ensinar e aprender língua estrangeira nas diferentes idades: reflexões para professores e formadores. São Carlos: Editora Clara Luz, 20 I8. p. 237-255.

POLIZERA, A. A.; ALVES, T. M. B. Perfil da satisfação e função sexual de mulheres idosas. Fisioterapia e Movimento, Curitiba, v. 22, n. 2, p. I5I-I58, abr./jun. 2009.

RODRIGUES, C. F. C. et al. Atividade sexual, satisfação e qualidade de vida em pessoas idosas. Revista Eletrônica de Enfermagem, Goiânia, v. 21, n. 57, p. I-9, 2019.

RODRIGUES, L. R. et al. Análise do comportamento sexual de idosas atendidas em um ambulatório de ginecologia. Revista Brasileira de Geriatria e Gerontologia, Rio de Janeiro, v. 21 , n. 6 , p. $724-730,2018$.

SALGADO, C. D. S. Mulher idosa: a feminização da velhice. Estudos Interdisciplinares sobre o Envelhecimento, Porto Alegre, v. 4, p. 7-19, 2002.

SATHYANARAYANA, R.; ABHINAV, T. S. M. Clinical Practice Guidelines for management of Sexual Disorders in Elderly. Indian Journal of Psychiatry, v. 60, n. 3, 2018.

SIQUEIRA, P. Terceira idade e sexualidade: um encontro possível? Fragmentos de Cultura, v. I7, 2017.

SOARES, F. A. Envelhecimento humano e atividade sexual. Revista Digital, Buenos Aires, n. 135, 2009. 
TAVARES, D. M. S.; CÔRTES, R. M.; DIAS, F.

A. Qualidade de vida e comorbidades entre os idosos diabéticos. Revista de Enfermagem da UERJ. v. I8, p. 97-103, 2010.

VIEIRA, S. et al. A vivência da sexualidade saudável nos idosos: o contributo do enfermeiro. Revista de Ciências da Saúde da ESSCVP, v. 6, jul. 2014. 